\title{
Emergent Calabi-Yau Geometry
}

\author{
Hirosi Ooguri ${ }^{1,2}$ and Masahito Yamazaki ${ }^{1,2,3}$ \\ ${ }^{1}$ California Institute of Technology, Pasadena, California 91125, USA \\ ${ }^{2}$ Institute for the Physics and Mathematics of the Universe, University of Tokyo, Kashiwa, Chiba 277-8586, Japan \\ ${ }^{3}$ Department of Physics, University of Tokyo, Hongo 7-3-1, Tokyo 113-0033, Japan
}

(Received 27 February 2009; published 21 April 2009)

\begin{abstract}
We show how the smooth geometry of Calabi-Yau manifolds emerges from the thermodynamic limit of the statistical mechanical model of crystal melting defined in our previous paper. In particular, the thermodynamic partition function of molten crystals is shown to be equal to the classical limit of the partition function of the topological string theory by relating the Ronkin function of the characteristic polynomial of the crystal melting model to the holomorphic 3-form on the corresponding Calabi-Yau manifold.
\end{abstract}

DOI: 10.1103/PhysRevLett.102.161601

PACS numbers: 11.25.Uv, 11.25.Mj

Introduction.-Stringy effects and quantum gravity effects are expected to modify our concept of space and time, and understanding such effects quantitatively is an important step in the exploration of physics at the Planck scale. In the perturbative string theory, where the string coupling constant $g_{s}$ is assumed to be small, the string length is longer than the Planck length. In this regime, stringy corrections to spacetime geometry kicks in first and quantum gravity effects are obscured. In the context of the topological string theory on $\mathbf{C}^{3}$, it was pointed out in Ref. [1] that the partition function can be resummed, and the result can be expressed in terms of a statistical model of crystal melting for large $g_{s}$. In our previous paper [2], we generalized this construction to an arbitrary toric CalabiYau threefold. In the crystal melting model, the Calabi-Yau geometry is discretized, and each atom of the crystal can be regarded as a fundamental unit of the geometry. In this Letter, we will show how the smooth Calabi-Yau geometry emerges from the discrete structure of the crystal melting model in the thermodynamic limit, where $g_{s} \rightarrow 0$. The topological string theory is relevant for counting microstates of black holes in the superstring theory [3], and we expect that our result sheds some light on the quantum nature of spacetime in the superstring theory also.

A toric Calabi-Yau 3-fold $M$ is a Kähler quotient of $\mathbf{C}^{F+3}$ by $U(1)^{\otimes F}$, and its mirror manifold $\tilde{M}$ is defined by the polynomial equation $[4,5]$,

$$
u v+P(z, w)=0, \quad(u, v) \in \mathbf{C}, \quad(z, w) \in \mathbf{C}^{\times} .
$$

Here $P(z, w)$ is a Newton polynomial of the form,

$$
P(z, w)=\sum_{i=1}^{F+3} c_{i}(t) z^{n_{i}} w^{m_{i}},
$$

and $c_{i}(t)$ 's are functions of the Kähler moduli $t$ of the original toric 3 -fold $M$. The exponents $\left(n_{i}, m_{i}\right) \in \mathbf{Z}^{2}$ correspond to lattice points of the toric diagram. For example, for the mirror of $\mathcal{O}(-1)+\mathcal{O}(-1)$ bundle over $\mathbf{C} P^{1}$, $P(z, w)$ is given by

$$
P(z, w)=1+z+w+e^{t} z w .
$$

In this Letter, we will show that the Newton polynomial $P(z, w)$ for the mirror of a Calabi-Yau manifold is identical to the characteristic polynomial of the corresponding dimer model, which is the partition function of the model on a torus. The relation between $P(z, w)$ and the characteristic polynomial had been discussed earlier in [6]. Here, we will prove their precise equality including the dependence on the moduli $t$.

The dimer model enters into our discussion because of its relevance to counting of D-brane bound states. Consider D0 and D2 branes with a single D6 brane on $M$. The low energy effective theory is a supersymmetric quiver quantum mechanics characterized by a quiver diagram on $T^{2}$ [6,7]. In our previous paper [2], we showed that the dimer model defined in Refs. [8,9] gives a generating function of the Witten indices for bound states in quiver quantum mechanics [10].

We also constructed a statistical model of crystal melting equivalent to the dimer model. The crystal consists of atoms of different types, each of which corresponds to a node of the quiver diagram for the quantum mechanics, and the chemical bonds between the atoms are dictated by edges of the diagram. The quiver diagram is drawn on $T^{2}$. To construct the initial configuration of the crystal, we start with the universal covering of the diagram over the plane and pile up atoms on nodes following the rules prescribed in Ref. [2]. This initial configuration corresponds to a single D6 brane with no D0 and D2 charges. Removing atoms from the crystal generates bound states with nonzero D0 and D2 charges. Such molten crystal configurations are in one-to-one correspondence with perfect matchings of the dimer model defined in Ref. [9], as shown in the Appendix of Ref. [2]. 
It is reasonable to expect that a classical geometric picture emerges in the limit of large D0 and D2 charges since it can represent a large black hole in the superstring theory. The corresponding thermodynamic limit in the dimer model was studied in Ref. [11]. In this Letter, we will show that the partition function of the dimer model evaluated in the thermodynamic limit is equal to the genus-0 limit of the partition function of the topological string theory on $M$. The dimer model has been formulated to describe the noncommutative Donaldson-Thomas theory $[8,9]$, while the topological string theory for a general toric Calabi-Yau manifold is equivalent to the commutative Donaldson-Thomas theory [12], as shown by [13]. A relation of our results to the wall-crossing formula [14-17] between the noncommutative and commutative Donaldson-Thomas theories is currently under investigation.

The emergence of Calabi-Yau geometry from the thermodynamic limit has been observed in Ref. [1] in the case of $\mathbf{C}^{3}$. In this Letter, we make the connection sharper and more explicit by showing the direct connection between the partition functions of the crystal melting model and the topological theory for a general toric Calabi-Yau 3-fold.

Thermodynamic limit of the crystal melting model.-The main object of study in this Letter is the partition function,

$$
Z=\sum_{q_{0}, q_{A}} \Omega\left(q_{0}, q_{A}\right) e^{-g_{s} q_{0}-t^{A} q_{A}},
$$

where $\Omega\left(q_{0}, q_{A}\right)$ is the Witten index for bound states of $q_{0}$ D0 branes and $q_{A}$ D2 branes on the $A$ th 2-cycle $(A=$ $1, \ldots, \operatorname{dim} H_{2}(M)$ ) with a single D6 brane on a toric Calabi-Yau manifold. According to the dictionary in Ref. [2], the Witten index is equal (up to a sign) to the number of molten crystal configurations where $q_{0}$ is the total number of atoms removed, whereas the relative numbers of different types of atoms removed from the crystal are specified by $q_{A}$ 's. Later we will identify $g_{s}$ as the topological string coupling constant and $t^{A}$ as the Kähler moduli of the toric Calabi-Yau manifold $M$.

The behavior of $Z$ for $g_{s} \rightarrow 0$ can be evaluated by using the result of Ref. [11]. Consider a finite covering the original quiver diagram, $N$ times in one direction and $N$ times in another direction on $T^{2} . N$ is introduced as an infrared regulator, and we will take $N \rightarrow \infty$ at the end of the computation so that we have the dimer model on the plane $\mathbf{R}^{2}$. The surface of the crystal is determined by the height function $h$ over the plane. To define $h$, we start with the canonical perfect matching $m_{0}$ of the dimer model corresponding to the initial crystal configuration with no D0 or D2 charges. For any other perfect matching $m$, the superposition of $m_{0}$ and $m$ gives a set of closed loops on the dimer graph. If $m$ corresponds to a bound state with finite D0 and D2 charges, $m$ and $m_{0}$ differ only in a finite region on the graph. The function $h_{i}(i$ : node of the $N \times N$ cover of the quiver) is defined so that it is 0 far away from the region where $m$ and $m_{0}$ differ, and it increases by 1 every time we cross a closed loop as we move inside of the region. The corresponding molten crystal configuration is obtained by removing $h_{i}$ atoms of the initial crystal over the node $i$. In particular,

$$
\sum_{i} h_{i}=q_{0} .
$$

To take the thermodynamic limit, it is useful to introduce the Cartesian coordinates $(x, y), 0 \leq x, y \leq 1$, on the $N \times$ $N$ covering of $T^{2}$. In the limit where $g_{s} \ll 1$ and $1 \ll N$, the height $h_{i}$ becomes a smooth function $h(x, y)$. We rescale the height function by the factor of $1 / N$ so that

$$
N^{2} \int_{0}^{1} d x d y h(x, y)=\frac{q_{0}}{N},
$$

to take into account the large $N$ scaling of the partition function discussed in Ref. [18] and quoted as Theorem 2.1 in Ref. [11]. The statistical weight in the thermodynamic limit is given by an integral of a surface tension $\sigma(\partial h)$, which is a function of the gradient of $h$, as [19]

$$
Z \sim \exp \left[N^{2} \max _{h} \int_{0}^{1} d x d y\left[-\sigma(\partial h)-g_{s} N h(x, y)\right]\right] .
$$

The integral of $g_{s} N h(x, y)$ in the exponent comes from the weight factor $e^{-g_{s} q_{0}}$ in (4), and we used (6). In the thermodynamic limit, we look for a height function $h(x, y)$ which maximize the exponent.

To derive the macroscopic surface tension from the microscopic crystal melting model, we first define a characteristic polynomial $\tilde{P}(z, w)$ as the sum of perfect matchings with weights assigned to edges of the dimer model on $T^{2}$ [11]. We then define its Ronkin function $R(x, y)$ by

$$
R(x, y)=\int_{0}^{2 \pi} \ln \tilde{P}\left(e^{x+i \theta}, e^{y+i \phi}\right) \frac{d \theta d \phi}{(2 \pi)^{2}} .
$$

According to Theorem 3.6 of Ref. [11], the surface tension $\sigma\left(\partial_{x} h, \partial_{y} h\right)$ is the Legendre transform of the Ronkin function with respect to $(s, t)=\left(\partial_{x} h, \partial_{y} h\right)$ as

$$
R(x, y)=\max _{s, t}[-\sigma(s, t)+x s+y t] .
$$

The first step in relating the dimer model to the topological string theory is to show that the characteristic polynomial $\tilde{P}(z, w)$ of the dimer model is equal to the Newton polynomial $P(z, w)$ for the mirror Calabi-Yau manifold (1),

$$
\tilde{P}(z, w)=P(z, w) .
$$

According to Ref. [20], there is a one-to-one correspondence between perfect matchings of the dimer model on $T^{2}$ and bifundamental fields of the gauged linear sigma model appearing in the Kähler quotient construction of the toric Calabi-Yau manifold $M$. They are then related, by change of variables described in Ref. [20], to lattice points of the toric diagram and to terms $z^{n_{i}} w^{m_{i}}$ in the Newton polyno- 
mial (1). This shows that there is a one-to-one correspondence between terms in $P(z, w)$ and $\tilde{P}(z, w)$, as pointed out by [6]. Furthermore we can show that their coefficients agree. The Kähler moduli of $M$ are the Fayet-Iliopoulos (FI) parameters of the quiver quantum mechanics. According to the dictionary, between quiver gauge theories and dimer models [7], FI parameters are associated with nodes of the quiver diagram, or equivalently to faces of the dimer model. Thus, we can identify the FI parameters with the magnetic fluxes through faces of the dimer model, which parametrize the energy of each perfect matching of the dimer model [11]. Each perfect matching appears in $\tilde{P}(z, w)$ with the weight given by an exponential of the fluxes. On the other hand, the Newton polynomial is a sum of $z^{n_{i}} w^{m_{i}}$, each of which corresponds to a lattice point of the toric diagram and is weighted by an exponential of the Kähler moduli $t$ [4]. We have verified that the weight for perfect matchings and the weight for lattice points of the toric diagram agree, and this proves the identity (10).

Combining (7) and (9) and discarding a term in total derivative in $(x, y)$, which is justified by the subtraction of the linear piece in $R(x, y)$ discussed in the next paragraph, we find

$$
Z \sim \exp \left[N^{2} \int d x d y R\left(\frac{g_{s} N}{2} x, \frac{g_{s} N}{2} y\right)\right] .
$$

By rescaling $(x, y)$ by the factor of $g N / 2$, this becomes

$$
Z \sim \exp \left[\frac{4}{g_{s}^{2}} \int d x d y R(x, y)\right] .
$$

Note that the $N$ dependence has disappeared except that the range of the $(x, y)$ integral has been rescaled by the factor of $g N / 2$. For $N \rightarrow \infty$ with small but fixed $g_{s}$, we have an integral over the whole $(x, y)$ plane.

The integral (12) in the large $N$ limit is divergent. To identify and subtract the divergent part, it is convenient to introduce the concept of the amoeba [21] which is a subset of $\mathbf{R}^{2}$ defined by,

$$
\begin{aligned}
& \text { Amoeba }=\left\{(x, y) \in \mathbf{R}^{2} P\left(e^{x+i \theta}, e^{y+i \phi}\right)=0\right. \\
& \text { for some }(\theta, \phi)\} .
\end{aligned}
$$

In the thermodynamic limit, the amoeba corresponds to the liquid phase of the crystal [11]. If there are no interior points in the toric diagram, the complement of the amoeba is the solid phase, where the crystal retains its original shape. There, the Ronkin function $R(x, y)$ is linear [24]. If there are interior lattice points in the toric diagram, the amoeba acquires holes, inside of which are in the gas phase, where the Ronkin function is again linear but the slope of the crystal surface is different from the original one. The integral (12) becomes finite if we subtract the linear piece of the Ronkin function in the solid phase so that the partition function is normalized to be 1 for the initial crystal configuration.
Topological string at genus 0.-Our next task is to compute the genus-0 topological string partition function $\mathcal{F}_{0}$ of the toric Calabi-Yau manifold $M$ and compare it with the thermodynamic limit of the partition function of the crystal melting model (12). For this purpose, it is convenient to use the mirror Calabi-Yau manifold $\tilde{M}$ defined by the Eq. (1) since $\mathcal{F}_{0}$ can be evaluated by the classical period integral as,

$$
\mathcal{F}_{0}=\int_{\beta_{0}} \Omega,
$$

where $\Omega$ is the holomorphic 3 -form on the mirror, and $\beta_{0}$ is the Lagrangian 3-cycle which is the mirror of the 6-cycle filling the entire toric Calabi-Yau manifold.

According to the microscopic derivation of the mirror symmetry by Hori and Vafa [4], the sigma model on the toric Calabi-Yau manifold is equivalent to the LandauGinzburg model with the superpotential

$$
W(u, x, y)=e^{u} P\left(e^{x}, e^{y}\right) .
$$

It was shown in Ref. [5] that an integral of $e^{W}$ in a threedimensional subspace of the $(u, x, y)$ plane can be transformed into a period integral of the holomorphic 3-form $\Omega$ on the mirror Calabi-Yau manifold. Thus, we should be able to evaluate (14) as an integral of $e^{W}$. To do so, we need to identify the contour of the integral.

Since $g_{s}$ and $t^{A}$ 's in (4) are taken to be real in the dimer model, the Newton polynomial $P(x, y)$ in our case is with real coefficients. The mirror manifold (1) has the complex conjugation involution, and thus the fixed point set is a natural candidate for $\beta_{0}$. In fact, following the mirror symmetry transformation as described in Ref. [4], we find that the 6-cycle of the original toric Calabi-Yau manifold $M$ corresponds to the real section in the $(u, x, y)$ space in the mirror $\tilde{M}$. Thus, we find

$$
\begin{aligned}
\mathcal{F}_{0} & =\int_{0}^{\infty} d u \int_{-\infty}^{\infty} d x d y e^{e^{u} P\left(e^{x}, e^{y}\right)} \\
& =-\int d x d y \ln P\left(e^{x}, e^{y}\right) .
\end{aligned}
$$

The divergent part of the integral in the $(x, y)$ plane can be removed by subtracting a linear term in $\ln P\left(e^{x}, e^{y}\right)$ for $x$, $y \rightarrow \infty$ as we did for (12). The integral (16) is almost equal to the exponent of the partition function (12) of the crystal melting model, except that we do not have the averaging over the phases $(\theta, \phi)$ to define the Ronkin function as in (8). It turns out that the integral over $(x, y)$ in (16) removes the dependence on $(\theta, \phi)$, and thus the averaging process is not necessary.

To see this, let us define a generalization of $\mathcal{F}_{0}$ for an integral of $(x, y)$ with arbitrary phases as

$$
\mathcal{F}(\theta, \phi)=\int d x d y \ln P\left(e^{x+i \theta}, e^{y+i \theta}\right) .
$$

Taking derivatives of $\mathcal{F}$ with respect to $(\theta, \phi)$, we find the 
integrand becomes a total derivative in $(x, y)$ as in

$$
\begin{aligned}
\left(\alpha \frac{\partial}{\partial \theta}+\beta \frac{\partial}{\partial \phi}\right) \mathcal{F}= & \int d x d y i\left(\alpha \frac{\partial}{\partial x}+\beta \frac{\partial}{\partial y}\right) \\
& \times \ln P\left(e^{x+i \theta}, e^{y+i \phi}\right) .
\end{aligned}
$$

If we choose $(\alpha, \beta)$ so that it is not in the direction of the tentacles of the amoeba (13), the boundary term is removed by the regularization and we find $\left(\alpha \partial_{\theta}+\beta \partial_{\phi}\right) \mathcal{F}=0$. Since $\alpha, \beta$ is arbitrary except in the direction of the tentacles of the amoeba, $\mathcal{F}$ is independent of $(\theta, \phi)$ and agrees with its average. Namely,

$$
\mathcal{F}_{0}=-\int d x d y \ln P(x, y)=-\int d x d y R(x, y) .
$$

Thus, we found that the thermodynamic limit of the partition function of the crystal melting model given by (12) is equal to $\exp \left(-\frac{4}{g_{s}^{2}} \mathcal{F}_{0}\right)$, which is the genus-0 partition function of the topological string theory. This is what we wanted to show.

We would like to thank Alexei Borodin, Kentaro Hori, Kentaro Nagao, and Andrei Okounkov for stimulating discussions. We thank Mina Aganagic for her comment on the earlier version of this Letter. This work is supported in part by DOE Grant No. DE-FG03-92-ER40701 and by the World Premier International Research Center Initiative of MEXT of Japan. H.O. is also supported in part by a Grant-in-Aid for Scientific Research (C) 20540256 of JSPS and by the Kavli Foundation. M. Y. is also supported in part by JSPS and GCOE for Phys. Sci. Frontier of MEXT of Japan.

[1] A. Okounkov, N. Reshetikhin, and C. Vafa, arXiv:hep-th/ 0309208.

[2] H. Ooguri and M. Yamazaki, arXiv:0811.2801 [Comm. Math. Phys. (to be published)].
[3] H. Ooguri, A. Strominger, and C. Vafa, Phys. Rev. D 70, 106007 (2004).

[4] K. Hori and C. Vafa, arXiv:hep-th/0002222.

[5] K. Hori, A. Iqbal, and C. Vafa, arXiv:hep-th/0005247.

[6] B. Feng, Y.H. He, K. D. Kennaway, and C. Vafa, Adv. Theor. Math. Phys. 12, 3 (2008).

[7] A. Hanany and K. D. Kennaway, arXiv:hep-th/0503149; S. Franco, A. Hanany, K. D. Kennaway, D. Vegh, and B. Wecht, J. High Energy Phys. 01 (2006) 096; S. Franco, A. Hanany, D. Martelli, J. Sparks, D. Vegh, and B. Wecht, J. High Energy Phys. 01 (2006) 128; K. Ueda and M. Yamazaki, arXiv:math.AG/0703267.

[8] B. Szendröi, Geom. Topol. 12, 1171 (2008).

[9] S. Mozgovoy and M. Reineke, arXiv:0809.0117.

[10] See, K. Larjo, arXiv:0902.0614, for subsequent developments.

[11] R. Kenyon, A. Okounkov, and S. Sheffield, arXiv:math$\mathrm{ph} / 0311005$.

[12] S. K. Donaldson and R.P. Thomas, in The Geometric Universe: Science, Geometry and The Work of Roger Penrose (Oxford University Press, Oxford, 1998); R.P. Thomas, J. Diff. Geom. 54, 367 (2000).

[13] D. Maulik, A. Oblomkov, A. Okounkov, and R. Pandharipande, arXiv:0809.3976.

[14] M. Kontsevich and Y. Soibelman, arXiv:0811.2435v1.

[15] K. Nagao and H. Nakajima, arXiv:0809.2992.

[16] K. Nagao, arXiv:0809.2994.

[17] D. L. Jafferis and G. W. Moore, arXiv:0810.4909; W. Y. Chuang and D. L. Jafferis, arXiv:0810.5072.

[18] S. Sheffield, Ph.D. thesis, Stanford University, 2003.

[19] Here we only show the $g_{s}$ dependence explicitly and the dependence on the Kähler moduli $t^{A}$ is in $\sigma$.

[20] S. Franco and D. Vegh, J. High Energy Phys. 11 (2006) 054.

[21] See Ref. [22] for a survey of aspects of amoebae, and Ref. [23] for their uses in other aspects of gauge theories.

[22] G. Mikhalkin, arXiv:math.AG/0108225.

[23] T. Maeda and T. Nakatsu, Int. J. Mod. Phys. A 22, 937 (2007); T. Fujimori, M. Nitta, K. Ohta, N. Sakai, and M. Yamazaki, Phys. Rev. D 78, 105004 (2008).

[24] L. I. Ronkin, in Complex Analysis in Modern Mathematics (Russian) (FAZIS, Moscow, 2001), pp. 239-251; M. Passare and H. Rullgård, Duke Math. J. 121, 481 (2004). 